\title{
Gamma Hydroxy Butyric Acid and Sodium Oxybate Used to Treat Posttraumatic Stress Disorder
}

\section{To the Editor:}

September 12, 2007

I write this case report to discuss the potential benefit of using sodium oxybate to treat posttraumatic stress disorder (PTSD). Sodium oxybate is currently Food and Drug Administation approved to treat excessive daytime sleepiness due to narcolepsy. ${ }^{1}$

In contrast to stimulants, which increase norepinephrine and dopamine activity, and modafinil, which likely increases histamine activity to improve alertness, gamma hydroxy butyric acid (GHB), and likely sodium oxybate's, mechanism of action may include the induction of deep, more restorative sleep through $\gamma$-aminobutyric acid-B (GABA-B) receptor agonism. Allopragnanolone and allotetrahydrodeoxycorticosterone are neurosteroids that are enhanced after GHB dosing, and these allosteric modulators increase GABA-A receptor activity lending to hypnotic effects as well.

Norepinephrine activity is dampened after GHB dosing, which may dampen arousal and allow better sleep. When GHB washout occurs there is a robust release of norepinephrine which may account for daytime alerting effects. At low doses, dopamine activity is lessened, which fosters an ultimate increase in synthesis and availability of dopamine in the substantia nigra and mesocortical pathways. This allows improved frontal lobe function with improved wakefulness and attention. Finally, serotonin metabolism also may increase in the mesolimbic system and the endogenous opiods, dynorphin and enkaphalin, may increase. ${ }^{2}$ This complicated mechanism of action is suggestive of an agent that may help improve sleep, wakefulness, and lessen anxiety symptoms as well.

\section{CASE REPORT}

Mr. Y is a married 36-year-old with one child and is gainfully employed in the security field. He was born in the eastern United States and moved to the West Coast around 11 years of age. He reports that his upbringing was largely uneventful in regards to trauma and that his basic needs were met. He reported academically that he always did well in most of his classes. $\mathrm{He}$ states he was a well-rounded individual in regards to friendships and social activities.

In the late 1980s he joined the Armed Forces at an early age with the permission of his parents. The patient reports that he was involved in a training accident where the vehicle he was in had an accident. As a result, he was severely injured and was flown by helicopter to the nearest trauma center. During the trauma and while he waited for the MEDEVAC he was witness to the death and dismemberment of his fellow soldiers. He sustained multiple fractures involving his face and extremities, had upper and lower back injuries, and his right shoulder and knees were injured.

Shortly after this accident, the patient reports having survivor guilt and admitted to the following symptoms: re-living events such as nightmares, repression of memory about the event, emotional distancing and numbing, loss of interest in enjoyable things, estrangement from peers and relationships, marked insomnia, irritability outbursts, and hypervigilance.

Despite these psychological and physical symptoms, the patient recovered enough to regain activeduty status and then served in the initial Persian Gulf war where he saw active combat. He received specialized training (for example, sniper training). The patient reports clear traumatic events that were 
reactivating in regards to the aforementioned PTSD symptoms here as well during his tour.

Following this service, he applied for and enrolled in special forces training. During training he suffered various injuries, including a cerebral hemorrhage during a parachute training exercise and the rupture of a nasal sinus during a water exercise. However, he reported that these injuries did not reactivate his posttraumatic symptoms. Following this he returned to the reserves. During this time, $\sim 10$ years after he initially entered the Armed Services, he also worked civilian security and learned that some of his military colleagues had been killed in action. He reported that his PTSD symptoms were relatively under control despite this bad news. In fact, up until this time, he had not received any pharmacologic treatment and very little psychotherapy treatment, if any, because he was able to manage his symptoms and continue in his workplace.

The patient reported that things changed on September 11, 2001, when the terrorist attacks in New York City were televised. The patient actually reported complete reactivation and worsening of his posttraumatic symptoms when he viewed fooage on the Internet of people trapped in the towers jumping to their deaths. He had an immediate return of insomnia and other hyperarousal symptoms as well as a clear onset of full major depressive disorder symptoms. He had never suffered depressive symptoms prior to this. This is when he first sought care due to the acute and incapacitating symptoms of PTSD combined with major depressive disorder.

During this initial phase of diagnosis and active treatment he was tried on the following medications: quetiapine $300 \mathrm{mg} / \mathrm{day}$, citalopram 20-40 $\mathrm{mg} / \mathrm{day}$, clonazepam $2 \mathrm{mg} /$ day, bupropion 300 $\mathrm{mg} / \mathrm{day}$, and divalproex $1,000 \mathrm{mg} / \mathrm{day}$ to treat his depressive and anxiety symptoms. The patient reported minor relief at best. More of a problem were his complaints of adverse effects (increases in hyperarousal, heart palpitations, and worsening of insomnia with some agents). He was also faced with marked sedation, fatigue, overeating, and resultant obesity, where he gained upwards of 250 pounds. Given his dissatisfaction with these options, he discontinued his medications without any ill effect and the side effects were alleviated.

As he was discouraged with prescription medications' inability to help and an increased sideeffect burden, he turned to alternative medications. While visiting a health food store he asked ques- tions of the employee and ultimately tried a version of GHB. At the time of his initial use of this over-the-counter product it was legal and was used to promote better sleep, deeper sleep, and, in theory, an increase of growth hormone. This product was often used by professional weightlifters for this latter reason. The patient reported that, within days, he was sleeping remarkably better and over weeks much of his depression and posttraumatic anxiety symptoms were alleviated.

He returned to school and obtained an associate's degree in Spanish and ultimately a bachelors degree in international relations. Previously, when his PTSD, depressive symptoms, and side effects were active, he was unable to complete school. The patient found this particularly distressful as he is a student that had always scored within the " $99^{\text {th }}$ percentile" on many standardized scores. He considers his return to school and completion of his degrees a remarkable success which he attributes to the medications ability to treat his anxiety disorder symptoms. The patient was married in this time frame and more recently has had his first child. The patient feels his PTSD is at least $70 \%$ better, and he is more social and more focused. However, he does report some intrusive symptoms that are relatively easily controlled. He was dosing his GHB at 2 tablespoons at bedtime from 1999 to 2002 during this time period. He reported no daytime side effects from GHB. GHB was ultimately withdrawn from the market in 2000 due to controversy that it could be abused as a sedative-hypnotic or even a date-rape product. ${ }^{3}$ The patient reports when he was off his medication much of his depression and PTSD symptoms returned. More recently, he became aware of sodium oxybate, written under the prescription name "Xyrem", being used to treat the excessive sleepiness due to narcolepsy. The patient became aware that this prescription, a Drug Enforcement Agency Class III controlled drug, is chemically similar to GHB but, altogether safer than GHB. He petitioned his prescribers to allow him this drug, but despite his efforts, the controversy and lack of FDA approval for its use in treating either insomnia or PTSD, he was not allowed to take this medication. Finally, starting one-year ago a psychiatrist allowed him a prescription of sodium oxybate and he was titrated to a dose of $12 \mathrm{mg}$ at bedtime followed by $6 \mathrm{mg}$ four hours after falling asleep. The patient reports within a few days to a few weeks he returned to normal functioning without insomnia, PTSD, or depressive symptoms. The patient 
felt that the prescription provided the same relief as his previous over-the-counter GHB product. He denies any current side effects from his prescription of sodium oxybate.

\section{DISCUSSION}

This case series suggests that previous over-thecounter usage of GHB treated the patient's PTSD symptoms. He was forced to wash out of this agent and his symptoms returned. More recently, the offlabel use of sodium oxybate allowed for symptom resolution again. This subject served as his own control and suggests the biological activity of this type of agent may be useful for treating anxiety disorder. The GABA, norepinephrine, serotonin activity of other FDA antidepressants are often used to treat anxiety. Sodium oxybate may manipulate similar chemicals and also facilitate a reduction in anxiety symptoms as seen in this patient. This case is obviously limited in design and the patient's response could be attributable to placebo effect or the fluctuating nature of chronic anxiety. Pilot and definitive studies of sodium oxybate in the treatment of anxiety may be warranted.

\section{CONCLUSION}

Sodium oxybate was successful in resolving this treatment-resistant PTSD patient's symptoms. $\mathrm{He}$ is doing well in all spheres of life as a result.

Sincerely,

Thomas L. Schwartz, MD

\section{REFERENCES}

1. Robinson DM, Keating GM. Sodium oxybate: a review of its use in the management of narcolepsy. CNS Drugs. 2007,21:337-354.

2. Padri D, Black J. gamma-Hydroxybutyrate/sodium oxybate: neurobiology, and impact on sleep and wakefulness. CNS Drugs. 2006;20:993-1018.

3. Baker JC, Harris SL, Dyer JE. Experiences of gamma hydroxybutyrate (GHB) ingestion: a focus group study. J Psychoactive Drugs. 2007;39:115-129.

Dr. Schwartz is associate professor in the Department of Psychiatry at the State Univeristy of New York Upstate Medical University in Syracuse.

Disclosure: Dr. Schwartz has not received any funding from Jazz Pharmaceuticals in the last 2 years. Previously he had received a single research grant from this company.

Please send letters to the editor to: CNS Spectrums, c/o Eric Hollander, MD, 333 Hudson St., 7th Floor, New York, NY 10013; E-mail: vi@mblcommunications.com.

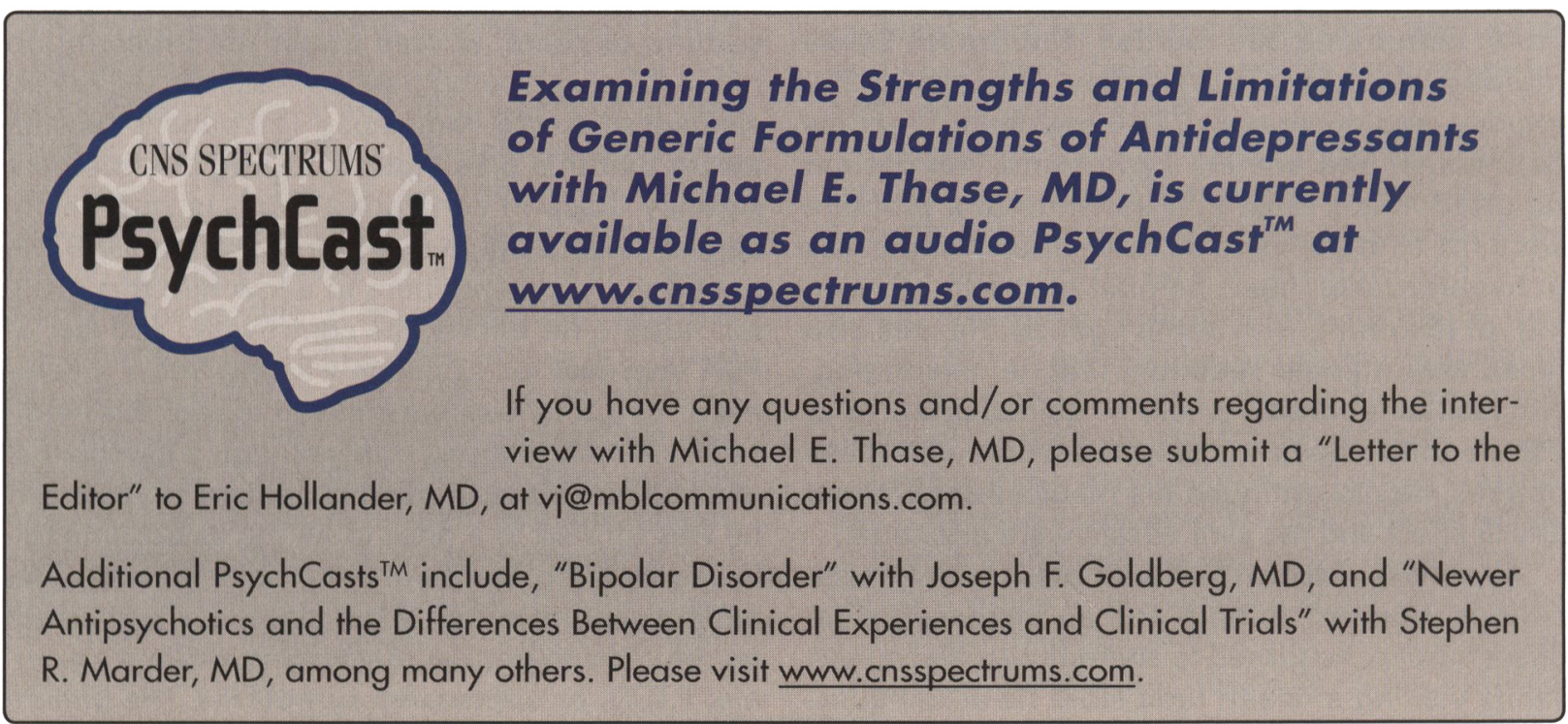


The things that may describe a patient with schizophrenia...

\author{
Delusions \\ Emotional withdrawal \\ Family history of high cholesterol \\ Disorganized behavior \\ ...can obscure the person
}




\section{ABILIFY Helps Reveal}

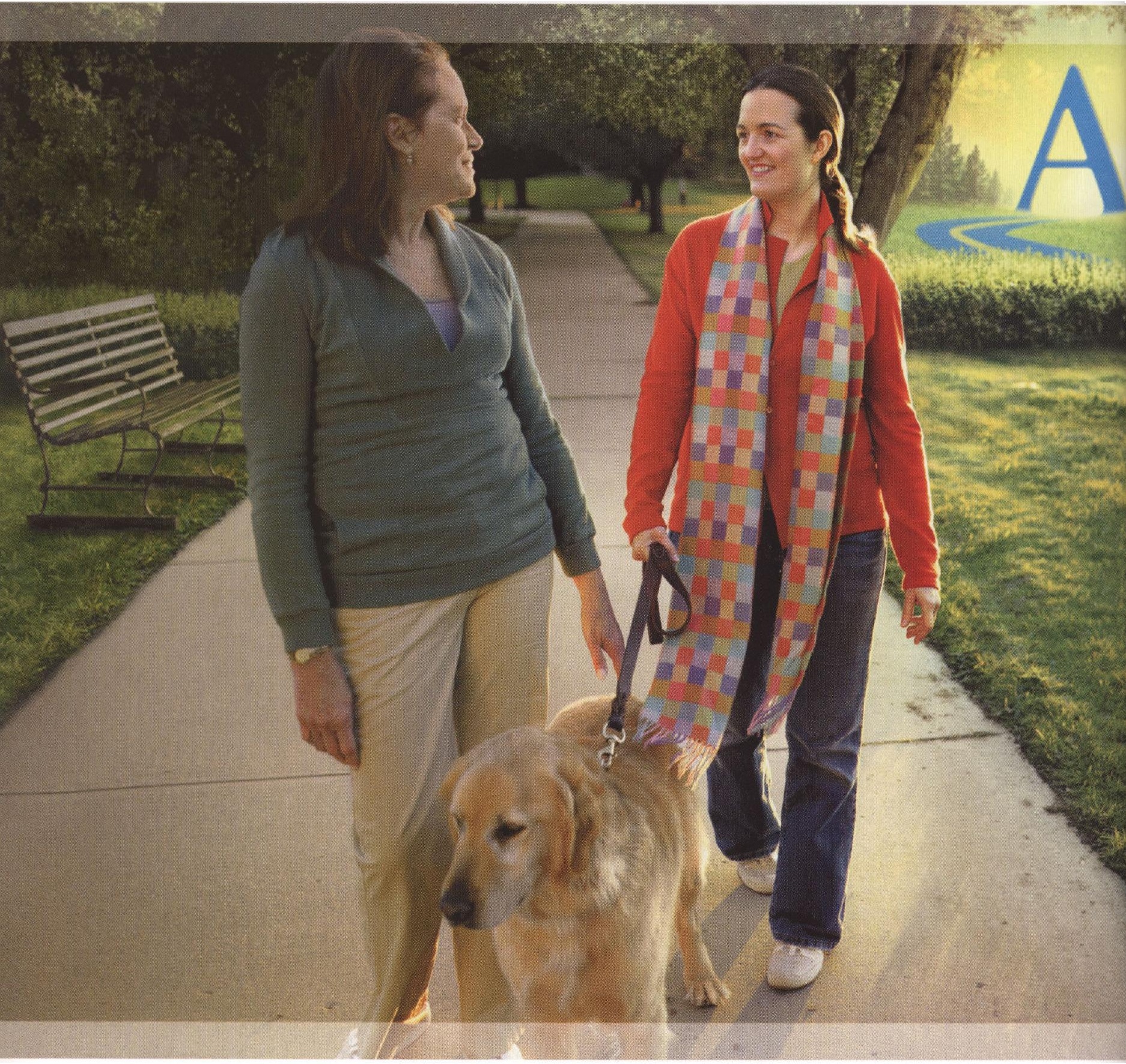

ABILIFY is indicated for the treatment of schizophrenia.

Elderly patients with dementia-related psychosis treated with atypical antipsychotic drugs are at an increased risk ( 1.6 to 1.7 times) of death compared to placebo ( $4.5 \%$ vs $2.6 \%$, respectively). ABILIFY is not approved for the treatment of patients with dementia-related psychosis. 


\section{The Person Within.}

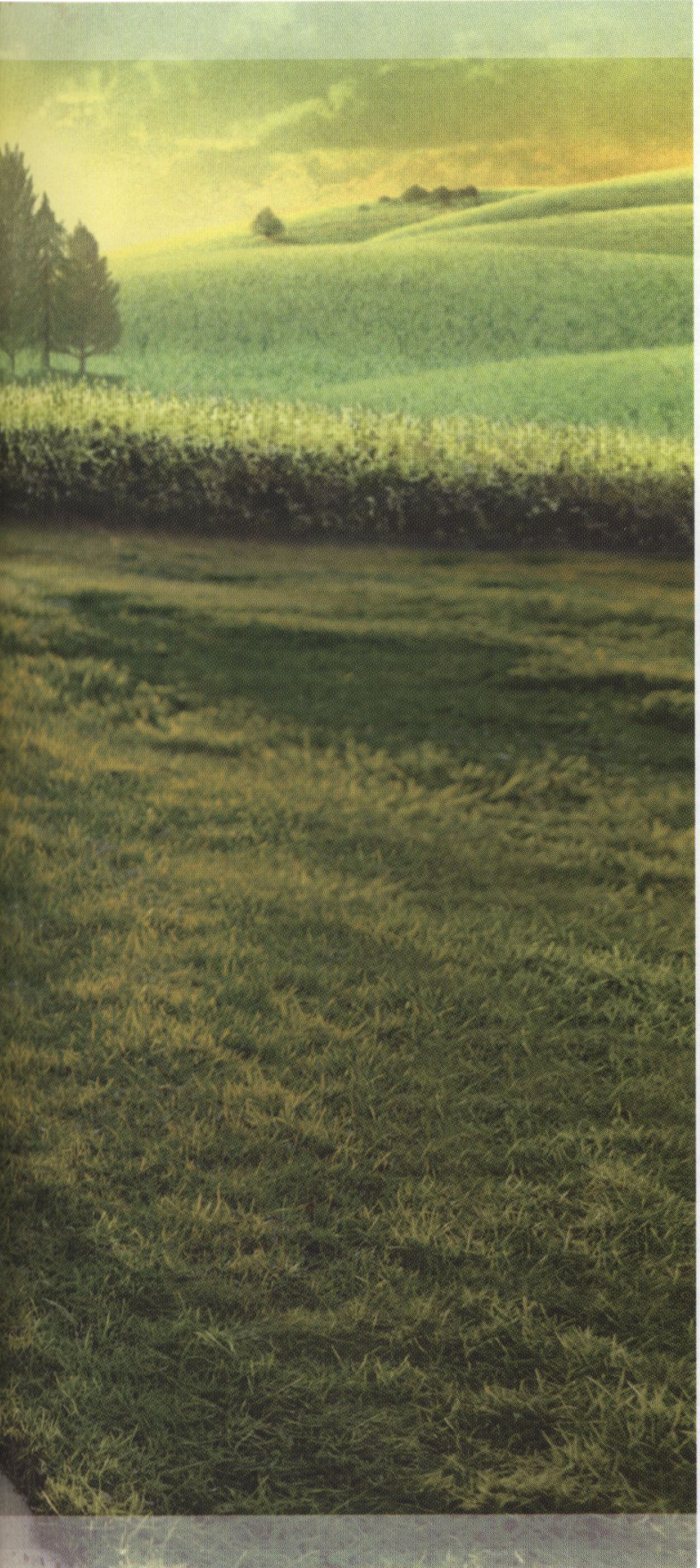

Meet Kristen, age 31. She is a patient with schizophrenia, but she's also an animal lover, daughter, and friend. She's so much more than her illness.

Do you have someone like Kristen in your practice?

ABILIFY significantly reduced positive and negative symptoms, as measured by PANSS ${ }^{\text {тм }}$ Total Score, at primary endpoint (Week 4) in a 4-week, double-blind, placebo-controlled trial in patients with schizophrenia.

In a long-term (26-week), placebocontrolled trial there were no medically important differences between the ABILIFY and placebo patients in the mean change from baseline in triglyceride, HDL, LDL, and total cholesterol measurements.

PANSS $^{\text {TM }}$ (Positive and Negative Syndrome Scale) is a trademark of Multi-Health Systems, Inc.

Please see IMPORTANT SAFETY INFORMATION, including Boxed WARNING, on following page.

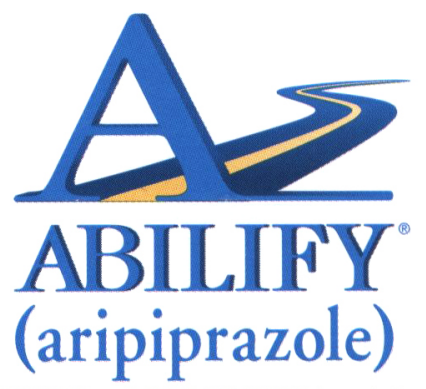


Elderly patients with dementia-related psychosis treated with atypical antipsychotic drugs are at an increased risk (1.6 to 1.7 times) of death compared to placebo ( $4.5 \%$ vs $2.6 \%$, respectively). ABILIFY is not approved for the treatment of patients with dementia-related psychosis (see Boxed WARNING).

Neuroleptic malignant syndrome (NMS)-As with all antipsychotic medications, a rare and potentially fatal condition known as NMS has been reported with ABILIFY. NMS can cause hyperpyrexia, muscle rigidity, diaphoresis, tachycardia, irregular pulse or blood pressure, cardiac dysrhythmia, and altered mental status. If signs and symptoms appear, immediate discontinuation is recommended

Tardive dyskinesia (TD)-The risk of developing TD and the potential for it to become irreversible may increase as the duration of treatment and the total cumulative dose increase. Prescribing should be consistent with the need to minimize TD. If signs and symptoms appear, discontinuation should be considered since TD may remit, partially or completely

- Cerebrovascular adverse events (eg, stroke, transient ischemic attack), including fatalities, have been reported at an increased incidence in clinical trials of elderly patients with dementia-related psychosis treated with ABILIFY
Hyperglycemia and diabetes mellitus-Hyperglycemia, in some cases associated with ketoacidosis, coma, or death, has been reported in patients treated with atypical antipsychotics including ABILIFY. Patients with diabetes should be monitored for worsening of glucose control; those with risk factors for diabetes should undergo baseline and periodic fasting blood glucose testing. Patients who develop symptoms of hyperglycemia should also undergo fasting blood glucose testing. There have been few reports of hyperglycemia with ABILIFY

Treatment-emergent adverse events reported with: ABILIFY Oral

In short-term trials of patients with schizophrenia (up to 6 weeks) or bipolar disorder (up to 3 weeks), the following were reported at an incidence $\geq 10 \%$ and greater than placebo, respectively: headache (30\% vs $25 \%)$, anxiety ( $20 \%$ vs $17 \%)$, insomnia ( $19 \%$ vs $14 \%)$, nausea ( $16 \%$ vs $12 \%)$, vomiting ( $12 \%$ vs $6 \%)$, dizziness ( $11 \%$ vs $8 \%)$, constipation ( $11 \%$ vs $7 \%)$, dyspepsia $(10 \%$ vs $8 \%)$, and akathisia ( $10 \%$ vs $4 \%)$.

\section{ABILIFY Injection}

In short-term ( 24 hour) trials, the following were reported at an incidence $\geq 5 \%$ and greater than placebo, respectively: headache $(12 \%$ vs $7 \%)$, nausea ( $9 \%$ vs $3 \%)$, dizziness ( $8 \%$ vs $5 \%)$, and somnolence $(7 \%$ vs $4 \%)$.

\title{
ABILIFY for Schizophrenia:
}

\section{Rapid control of agitation*}

Early $^{\dagger}$ and sustained positive and negative symptom control

Low incidence of somnolence/sedation ${ }^{\ddagger}$

Low mean weight change in clinical trials

- In a 52-week schizophrenia trial, weight change averaged $1 \mathrm{~kg}$ for ABILIFY-treated patients (BMI <23, $2.6 \mathrm{~kg}$; BMI 23 to 27, $1.4 \mathrm{~kg}$; BMI >27, -1.2 kg). The percentage of ABILIFY-treated patients with $\geq 7 \%$ increase in baseline body weight was $30 \%$ for those with BMI $<23,19 \%$ for those with BMI 23 to 27 , and $8 \%$ for those with BMI $>27$.

\section{Lipid profile comparable to placebo}

*With ABILIFY Injection at primary endpoint (2 hours). ABILIFY Injection is indicated for the treatment of agitation associated with schizophrenia.

${ }^{\dagger}$ As early as Week 1 through study endpoint (Week 4).

${ }^{\ddagger}$ ABILIFY $10 \%$, placebo $8 \%$.

Physicians who elect to use ABILIFY for extended periods should periodically re-evaluate the long-term usefulness of the drug for the individual patient.

Like other antipsychotics, ABILIFY may have the potential to impair judgment, thinking, or motor skills. Patients should not drive or operate hazardous machinery until they are certain ABILIFY does not affect them adversely.

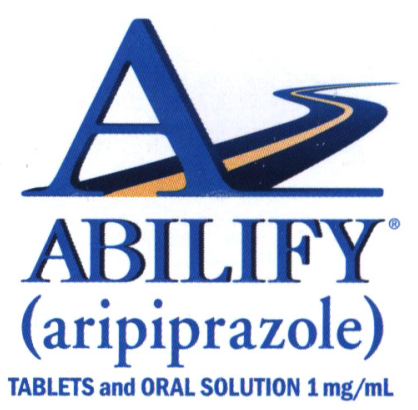

\section{HELP ILLUMINATE THE PERSON WITHIN}

\author{
Please see BRIEF SUMMARY OF FULL PRESCRIBING INFORMATION, \\ including Boxed WARNING, on adjacent pages.
}

Reference: 1. Potkin SG, Saha AR, Kujawa MJ, et al. Aripiprazole, an antipsychotic with a novel mechanism of action, and risperidone vs placebo in patients with schizoaffective disorder. Arch Gen Psychiatry. 2003;60:681-690. 
ABILIFY ${ }^{\circledR}$ (aripiprazole) TABLETS

Rx only

ABILIFY $^{\circledR}$ (aripiprazole) ORAL SOLUTION

ABILIFY ${ }^{\circledR}$ DISCMELT ${ }^{\mathrm{TM}}$ (aripiprazole) Orally Disintegrating Tablets

ABILIFY ${ }^{\circledR}$ (aripiprazole) INJECTION FOR INTRAMUSCULAR USE ONLY BRIEF SUMMARY: PLEASE CONSULT PACKAGE INSERT FOR COMPLETE PRESCRIBING INFORMATION.

INCREASED MORTALITY IN ELDERLY PATENTS WITH DEMENTIA-RELATED PSYCHOSIS
Elderty patients with dementia-rolated psychosis treated with atypical antipsychotic drugs are at an
increased risk of death compared to placebo. Analyses of seventeen placebo-controlled trials (modal
duration of 10 weaks) in these patients revealed a risk of death in the drug-treated patients of
between 1.6 to 1.7 tmes that seen in placebo-treated patients. Over the course of a typical to-week
controlled trial, the rate of death in drug-treated patients was about $4.5 \%$, compared to a rate of
about $2.6 \%$ in the placebo group. Although the causes of death were varied, most of the deaths
appeared to be elther cardlovascular (eg, heart failure, sudden death) or infectious (eg, pneumonia)
in nature. ABILIFY is not approved for the treatment of patients with dementia-related psychosis.

CONTRAINDICATIONS: Known hypersensitivity to aripiprazole

WARNINGS: Increased Mortality In Elderly Patients With Dementia-Related Psychosis - Elderly patients with dementla-related psychosis treated with atypical antipsychotic drugs are at an treatment of patients with dementia-related psychosis (see Boxed WARNiNG).

Neuroleptic Malignant Syndrome (NMS): Potentially fatal NMS has been reported in association with ition of antipsychotic drugs, including ABILIFY. Clinical manifestations of NMS are hyperpyrexia,

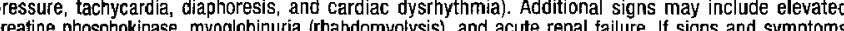
creatine phosphokinase, myoglobinuria (rhabdomyolysis), and acute renal failure. If signs and symptoms
appear, immediate discontinuation is recommended (see Full Prescribing Information for additional information on management of NMS). Patients requiring antipsychotic drug treatment after recovery from information on management of NMS). Patients requiring antipsychotic drug treatm
NMS should be carefully monitored since recurrences of NMS have been reported.

Tardlve Dyskinesia (TD): Potentially irreversible TD may develop in patients treated with antipsychotic drugs. Although the prevalence of TD appears to be highest among the elderly, especially elderly women, it is impossible to predict which patients are more likely to develop the syndrome. The risk of developing TD and the potential for it to become irreversible may increase as the duration of treatment and the total cumulative dose increase. Prescribing should be consistent with the need to minimize TD. If signs and
symptoms appear, discontinuation should be considered since TD may remit, partially or completely. symptoms appear, discontinuation should be considered since TD may remit, partialy or completely. Antipsychotic treatment, itself, may suppress (or partially suppress) the signs and symptoms of the syndrome and, thereby, may possible mask the underlying process. Chronic antipsychotic treatment should
generally be reserved for patients who suffer from a chronic illness that (1) is known to respond to antipsychotic drugs, and (2) for whom alternative, equally effective, but potentially less harmful treatments are not available or appropriate. The need for continued treatment should be reassessed periodically.

Cerebrovascular Adverse Events, Including Stroke, in Elderly Patients with Dementia-felated Psychosis: In placebo-controlied clinical studies (two flexible-dose and one fixed-dose study) of dementia-
related psychosis, there was an increased incidence of cerebrovascular adverse events (eg. stroke, transient related psychosis, there was an increased incidence of cerebrovascular adverse events (eg. stroke, transient
ischemic attack, including tatalities, in aripiprazole-treated patients. In the fixed-dose study, there was a ischemic attack), including tatalities, in aripiprazole-treated patients. In the fixed-dose stucty, there was a
statistically significant dose response relationship for cerebrovascular adverse events in patients treated with statistically significant dose response relationship for cerebrovascular adverse events in patients treated with
arpiprazole. ABILIFY is not approved for the treatment of patients with dementia-related pSychosis. (See also aripiprazole. ABILIFY is not approved for the treatment of patients with dementia-related
Boxed WARNING, WARNINGS and PRECAUTIONS in Full Prescribing Information)

Hyperglycemla and Diabetes Mollitus: Hyperglycemia, in some cases associated with ketoacidosis, hyperosmolar coma or death, has been reported in patients treated with atypical antipsychotics including ABLIFY. Assessmant of the relationship between atypical antipsychotic use and glucose abnormalities is complicated by the possibility of an increased background risk of dlabetes mellitus in patients with schizophrenia and the increasing incidence of diabetes mellitus in the general population. Patients diagnosed with diabetes who are started on atypical antipsychotics should be monitored regularly for worsening of glucose control;

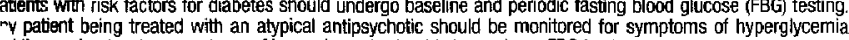
v patient being treated with an atypical antipsychotic should be monitored for sympt
$\mathrm{j}$ those who develop symptoms of hyperglycemia should also underge FBG testing.

\section{PRECAUTIONS: General:}

Orthostatic Hypotension: ABILIFY may be associated with orthostatic hypotension, perhaps due to its $a_{1}$-adrenergic receptor antagonism. The incidence of orthostatic hypotension-associated events from five short-term, placebo-controlled trials in schizophrenia $(n=926)$ on oral ABILIFY included: orthostatic hypotension $(1.9 \%)$, postural dizziness $(0.8 \%)$, and syncope $(0.6 \%)$. The incidence of orthostatic ABILIFY included: orthostatic hypotension $(0.7 \%)$, postural dizziness $(0.5 \%)$, and syncope $(0.3 \%)$. The Incidence of orthostatic hypotension-associated events from short-term, placebo-controlled trials in agitation associated with schizophrenia or b/polar mania $(n=501)$ on ABILIFY Injection included: orthostatic orthostatic change in blood pressure (defined as a decrease of at least $30 \mathrm{mmHa}$ in systolic biood pressure when changing from a supine to standing position) for aripiprazole was not statistically different from placebo in trials in patients with schizophrenia, bipolar mania, or agitation associated with schizophrenia or placebo in trials in patients with schizophrenia, bipolar mania, or agitation associated with schizophrenia or
bipolar mania. ABILIFY should be used with caution in patients with known cardiovascular disease (history bipolar mania. ABILIFY should be used with caution in patients with known cardiovascular disease (history
of myocardial infarction or ischemic heart disease, heart failure or conduction abnormalities), cerebrovascular disease, or conditions which would predispose patients to hypotension (dehydration, hypovolemia, and treatment with antihypertensive medications). If parenteral benzodiazepine therapy is deemed necessary in addition to ABILIF

Seizures: in short-term trials, seizures/convulsions occurred in $0.1 \%(1 / 926)$ of oral aripiprazole-treated patients with schizophrenia, in $0.3 \%$ (2/597) of oral aripiprazole-treated patients with bipolar mania, and in mania. Use cautiously in patients with a history of seizures or with conditions that lower the seizure threshold mania. Use cautiously in patients with a history of seizures or with conditions that lower the seizure thr

Potential for Cognitive and Motor Impairment: Despite the relatively modest Increased incidence of somnolence compared to placebo, ABILIFY, like other antipsychotics, may have the potential to impaif judgment, thinking, or motor skills. In short-term trials, somnolence (including sedation) was reported in $10 \%$ of patients with schizophrenia on oral ABILIFY compared to $8 \%$ of patients on placebo; $14 \%$ of with agitation associated with schizophrenia or bipolar mania on ABILIFY Injection compared to $6 \%$ of patients on placebo. Patients should be cautioned about operating hazardous machinery, including automabiles, until they are reasonably certain that therapy with ABILIFY does not affect them adversely. Body Iemperature Regulation: Disruption of body temperature regulation has been attributed to antipsychotic agents. Use appropriate care when prescribing aripiprazole for

Dysphagia: Esophageal dysmotility and aspiration have been associated with antipsychotic drug use, including ABILIFY. Aspiration pneumonia is a common cause of morbidity and mortality in patients with
advanced Alzheimer's disease. ABILIFY and other antipsychotic drugs should be used cautiously in patients advanced Alzheimer's disease.

Suicide: The possibility of a suicide attempt is inherent in psychotic illnesses and bipolar disorder, and close supervision of high-risk patients should accompany drug therapy. Prescriptions for ABILIFY should be

Use in Patients with Concomitant Illness: Clinical experience with ABILIFY in patients with certain concomitant systemic illnesses is limited. ABILIFY has not been evaluated or used to an

In three, 10-week, placebo-controlled studies of aripiprazole in elderly patients with psychosis associated with Alzheimer's disease $(n=938$, the treatment-emercent advise events that were reported at an incidence of $\geq 3 \%$-and. aripiprazole incidence at least twice that for placebo were lethargy, somnolence (including sedation), incontinence (primarily, urinary incontinence), excessive salivation, and lightheadedness. ABILIFY is
not approved for treatment of patients with dementia-related psychosis. If the prescriber elects to treat such enssive somnolence, vigilance should be exercised, particularly for the emergence of difficulty swallowing of Lacessive somnolence, which could predispose to accidental injury or aspiration (See Boxed WA
WARNALGS and CLINICAL PHARMACOLOGY: Special Populations in Full Prescribing Information).
Information for Patients: Physicians are advised to discuss the following issues with patients for whom they prescribe ABILIFY (aripiprazole) see fuss with patients taking aripiprazole:

Interference with Cognitive and Motor Performance: Patients should be cautioned about operating hazardous machinery, including automobiles, until they are reasonably certain that ABILIFY does not affect them adversely.

Pregnancy: Patients should be advised to notify their physician if they become pregnant or intend to Pregnant during therapy with ABILIFY.

Mursing: Patients should be advised not to breast-feed an infant if they are taking ABILIFY.

Concomitant Medication: Patients should be advised to inform their physicians if they are taking, or plan to take, any prescription or over-the-counter drugs, since there is a potential for interactions.

Heat Exposure and Dehydration: Patients should be advised regarding appropriate care in avoiding overheating and dehydration.

Phenylketonurics: Phenylalanine is a component of aspartame. Each ABILIFY DISCMELT orally disintegrating

Sugar Content Patients should be advised that each $\mathrm{mL}$ of ABILIFY oral solution contains $400 \mathrm{mg}$ of sucrose and $200 \mathrm{mg}$ of fructose.

Drug Interactions: Use caution when ABILFY is taken in combination with other centrally acting drugs and alcohol. ABiLIFY may enhance the effect of certain antihypertensive agents. ABILIFY is unlikely to CYP2B6, CYP2C8, CYP2C9, CYP2C19, or CYP2E 1 enzymes. $m$ vivo studies using 10 - to 30 -mg/day doses of aripiprazole had no significant effect on metabolism by CYP2D6 (dextromethorphan), CYP2CS (warfarin), CYP2C19 (omeprazole, wartarin), and CYP3A4 (dextromethorphan) substrates. No clinically Inducers of CYP3A4 (eg, carbamazepine) could cause an increase in aripiprazole clearance and lower blood levels. When a CYP3A4 inducer is added to ABILIFY, the dose of ABILIFY should be doubled. Additional dose increases should be based on clinical evaluation.

Carbamazepine: Coadministration of carbamazepine (200 mg BID) with ABILIFY (30 mg OD) resulted in an approximate $70 \%$ decrease in $C_{\max }$ and $A U C$ values of aripiprazole and its active metabolite, dehydro-

Inhibitors of CYP3A4 (eg, ketoconazole) or CYP2D6 (eg, quinidine, fluoxetine, or paroxetine) can inhibit the elimination of aripiprazole and cause increased blood levels. When a strong CYP3A4 or CYP2D6 inhibitor is added to ABILIFY, the dose of ABILIFY should be reduced to one-half of the usual dose. When the CYP3A4
CYP2D6 inhibitor is withdrawn from the combination therapy, the ABILIFY dose stould then be increased. Ketoconazoie: Coadministration of ketoconazole ( $200 \mathrm{mg} /$ day for 14 days) with a $15-\mathrm{mg}$ single dose of
ABILIFY increased the AUC of aripiprazole and its active metabolite by $63 \%$ and $77 \%$, respectively.

Quinidine: Coadministration of a 10-mg single dose of ABILIFY with quinidine (166 mg/day for 13 days aripiprazole, by $35 \%$.

Alcohol: There was no significant difference between aripiprazole coadministered with ethanol and placebo coadministered with ethanol on pertormance of gross motor skills or stimulus response in healthy subject
As with most psychoactive medications, patients should be advised to avoid alcohol while taking ABILIFY.

Carcinogenesis, Mutagenesis, Impairment of Fertility: Carcinogenesis: Carcinogenicity studies were 2 years in the diet at doses of $1,3,10$, and $30 \mathrm{mg} / \mathrm{kg} / \mathrm{day}$ to ICR mice and at $10,20,40,60 \mathrm{mg} / \mathrm{kg} / \mathrm{day}$ ( 3 to 19 times the maximum recommended human dose [MRHD] based on $\mathrm{mg} / \mathrm{m}^{2}$ ) to $\mathrm{SD}$ rats and 1,3 , and $10 \mathrm{mg} / \mathrm{kg} /$ day to $\mathrm{F} 344$ rats $\left(0.2\right.$ to 5 and 0.3 to 3 times the MRHD based on $\mathrm{mg} / \mathrm{m}^{2}$, respectively). In addition, $\mathrm{SD}$ rats were dosed orally for 2 years. Aripiprazole did not induce tumors in male mice or rats. In femaile mice, the incidences of pituitary gland adenomas and mammary gland adenocarcinomas and adenoacanthomas were increased at dietary doses of 3 to $30 \mathrm{mg} / \mathrm{kg} /$ day $(0.1$ to 0.9 times human exposur exposure at MRHD based on AUC and 3 times the MRHD based on $\left.\mathrm{m} / \mathrm{g}^{2}\right)$; and the incidences of adrenocortical carcinomas and combined adrenocortical adenomas/carcinomas were increased at an ora dose of $60 \mathrm{mg} / \mathrm{kg} / \mathrm{day}$ ( 14 times human exposure at MRHD based on AUC and 19 times the MRHD based on $\mathrm{mg} / \mathrm{m}^{2}$ ). These findings are considered to be prolactin-mediated. Increases in serum prolactin were prolactin was not increased in a 4 - and 13 -week dietary study in female rats. The relevance for human risk of prolactin-mediated endocrine tumors in radents is unknown. Mutagenesis: Aripiprazole and a metabolite $(2,3-\mathrm{DCPP})$ were clastogenic in the in vito chromosomal aberration assay in Chinese hamster lung (CHL)
cells, with and without metabolic activation. The metabolite, 2,3-DCPP, produced increases in numerical aberrations in the in vitro assay in CHL cells in the absence of metabolic activation. A positive response was obtained in the in vivo micronucleus assay in mice; however, the response was shown to be due to mechanism not considered relevant to humans. Impairment of Fertility: Female rats were treated with oral
doses of 2,6 , and $20 \mathrm{mg} / \mathrm{kg} /$ day $\left(0.6,2\right.$ and 6 times the MRHD on an $\mathrm{mg} / \mathrm{m}^{2}$ basis) of aripiprazole from 2 weeks prior to mating through day 7 of gestation. Estrus. cycle irregularities and increased corpora lute were seen at ail doses, but no impairment of fertility was seen. Increased pre-implantation loss was seen at
6 and $20 \mathrm{mg} / \mathrm{kg}$, and decreased fetal weight was seen at $20 \mathrm{mg} / \mathrm{kg}$. Male rats were treated with aral doses of 20,40 , and $60 \mathrm{mg} / \mathrm{kg} / \mathrm{day}\left(6,13\right.$, and 19 times the MRHD on an $\mathrm{mg} / \mathrm{m}^{2}$ basis of aripiprazole from 9 weeks prior to mating through mating. Disturbances in spermatogenesis were seen at $60 \mathrm{mg} / \mathrm{kg}$, and prostate atrophy was seen at 40 and $6.0 \mathrm{mg} / \mathrm{kg}$, but no impairment of fertility was seen

Pregnancy Category C: There are no adequate and well-controlled studies in pregnant women. Aripiprazole should be used in pregnancy only if the potential benefit justifies the potential risk to the etus. In animal studies,

Labor and Delivery: The effect of aripiprazole on labor and delivery in humans is unknown.

Nursing Mothers: Aripiprazole was excreted in milk of rats during lactation. It is not known whether aripiprazole or its metabolites are
aripiprazole should not breast-feed.

Pediatric Use: Safety and effectiveness in pediatric and adoliescent patients have not been established. Geriatric Use: Placebo-controlled studies of oral aripiprazole in schizophrenia or bipolar mania did not from younger subjects. There was no effect of age on the pharmacokinetics of a single 15-mg dose from younger subjects. There was no effect of age on the pharmacokinetics of a single $15-\mathrm{mg}$ dose o
aripiprazole. Aripiprazole clearance was decreased by $20 \%$ in elderly subjerts ( 265 years) compared to aripiprazole. Aripiprazole clearance was decreased by $20 \%$ in elderly subjects ( 265 years) compared to pharmacokinetic analysis in schizophrenia patients. Studies of elderly patients with psychosis associated with Alzheimer's disease have suggested that there may be a different tolerability profile in this
population compared to younger patients with schizophrenia. (See also Boxed WARNING, WARNINGS and PRECAUTIONS in Full Prescribing Intormation)

ADVERSE REACTIONS

Aripiprazole has been evaluated for safety in 8456 patlents who participated in multiple-duse, clinica trials in schizophrenia, bipolar mania, and dementia of the Alzheimer's type, and who had approximately injection patients treated with oral aripiprazole had at least 1 year of exposure.

Adverse Events Associated with Discontinuation of Treatment: Overall, there was little difference in the incidence of dliscontinuation due to adverse events in placebo-controlled oral aripiprazole trials (aripiprazole vs placebo: schizophrenia, $7 \%$ vs $9 \%$; bipolar mania, $11 \%$ vs vs $9 \%$; of in placebo-controlled intramuscular aripiprazole injection trials (aripiprazole injection, $0.8 \%$; placebo $0.5 \%$. The types of adverse

Commonly Observed Adverse Events: ( $25 \%$ incidence and at a rate at least twice the rate of placebo for ABILIFY vs placebo, respectively): In 4- to 6-week, placebo-controlled, schizophrenia trials (2 to $30 \mathrm{mg} / \mathrm{day}$ ) the one commonly observed adverse event associated with the use of oral aripiprazole was: akathisia $8 \%, 4 \%$. In 3-week, placebo-controlled, bipolar mania triais ( 15 or $30 \mathrm{mg} /$ day), the most common advers events associated with oral aripiprazole were: akathisia $(15 \%, 3 \%)$, constipation $(13 \%, 6 \%)$, sedation controlled trials of intramuscular aripiprazole injection for agitation associated with schizophrenia or bipola Adverse Events with an Incidence $\geq 2 \%$ in Oral Aripiprazole Trials: The following treatment-emergent 
events were reported at an incidence of $\geq 2 \%$ with oral aripiprazole (doses $\geq 2 \mathrm{mg} / \mathrm{d}$ ), and at a greate incidence with aripiprazole than with placebo in short-term placebo-controlled trials (aripiprazole $\mathrm{N}=1523$ nausea $(16 \%, 12 \%)$ vomiting $(12 \%, 6 \%$ dizziness $(11 \%, 8 \%)$, constipation $(11 \%, 7 \%)$ dyspepsia $(10 \%)$ $8 \%)$, akathisia $(10 \%, 4 \%)$, sedation $(7 \%, 4 \%)$, fatigue $(6 \%, 5 \%)$, extrapyramidal disorder $(6 \%, 4 \%)$, pharyngolaryngeal pain $(4 \%, 3 \%)$, $\{3 \%, 2 \%\}$, abdominal discomfort $\{3 \%, 2 \%)$, stomach discomfort $(3 \%, 2 \%)$, pain $(3 \%, 2 \%)$, vision blurred $(3 \%, 1 \%)$, salivary hypersecretion $(2 \%, 1 \%)$, peripheral edema $(2 \%, 1 \%)$, hypertension (including blood pressure increased) $(2 \%, 1 \%)$. The following events were reported by patients treated with oral aripiprazol with an incidence equal to or less than placebo: ciarrhea, toothache, upper abdominal pain, abdominal (percentage based on gender total), and rash.

Adverse Events with an Incidence $\mathbf{2 1 \%}$ in Intramuscular Aripiprazole Injection Trials: The following treatment-emergent events were reported at an incidence $z 1 \%$ with intramuscular aripiprazole injection doses $\geq 5.25 \mathrm{mg} / \mathrm{day}$ ) and at incidence greater than placebo in 24-hour, placebo-controlled trials placebo $\mathrm{N}=220$ ) in agitated patients with schizophrenia or bipolar mania, respectively, include: headache $(12 \%, 7 \%)$, nausea $(9 \%, 3 \%)$, dizziness $(8 \%, 5 \%)$, somnolence $(7 \%, 4 \%)$, dyspepsia $(1 \%,<1 \%)$, dry mouth $(1 \%,<1 \%)$, blood pressure increased $(1 \%,<1 \%)$, musculosketetal stifness $(1 \%,<1 \%)$. The following events were reported by patients treated with aripiprazole injection with

Dose-Related Adverse Events: Dose response relationships for the incidence of treatment-emergen adverse events were evaluated from four trials in patients with schizophrenia comparing various fixed doses $(2,5,10,15,20$, and $30 \mathrm{mg} / \mathrm{day}$ ) of oral aripiprazole to placebo. The one adverse event to have a
possible dose response relationship was somnolence (including sedation) which was most prominent at possible dose response relationship was somnolence (including sedation) which was most pr
the $30 \mathrm{mg} /$ day dose (piacebo, $7.1 \% ; 10 \mathrm{mg}, 8.5 \% ; 15 \mathrm{mg}, 8.7 \% ; 20 \mathrm{mg}, 7.5 \% ; 30 \mathrm{mg}, 12.6 \%$.

Extrapyramidal Symptoms: In the short-term, placebo-controlled trials of schizophrenia, the incidence of reported EPS-related events, excluding events related to akathisia was (oral aripiprazole $13 \%$, placebo $12 \%$ ) and the incidence of akathisia-related events was (oral aripiprazole $8 \%$, placebo $4 \%$ ). In the short term, placebo-controlled trials in bipolar mania, the incidence of reported EPS-related events, excluding events related to akathisia was (oral aripiprazole $15 \%$, placebo $8 \%$ ) and the incidence of akathisia-related events was (oral aripiprazole $15 \%$, placebo $4 \%$ ). In the placebo-controlled trials in patients with agitation associated with schizophrenia or bipolar mania, the incidence of reported EPS-related events excluding events related to akathisia was (aripiprazole injection $2 \%$,
related events was (aripiprazole injection $2 \%$, placebo $0 \%$.

Laboratory Test Abnormalities: A between group comparison for 3- to 6-week, placebo-controlled trials revealed no medically important differences between the aripiprazole and placebo groups in the proportions of patients experiencing potentially clinically significant changes in routine serum chemistry hematology, or urinalysis parameters. Similarly, there were no aripiprazole/placebo differences in the incidence of discontinuations for changes in serum chemistry, hematology, or urinalysis. In a long-term
(26-week) placebo-controlled trial there were no medically important differences between the aripiprazole and placebo patients in the mean change from baseline in prolactin, fasting glucose, triglyceride, HOL, $L D L$, and total cholesterol measurements.

Weight Gain: In 4- to 6-week trials in schizophrenia, there was a slight difference in mean weight gain between aripiprazole and placebo patients $(+0.7 \mathrm{~kg}$ vs. $-0.05 \mathrm{~kg}$, respectively), and also a difference in the placebo (3\%)]. In 3-week trlals in mania, the mean weight gain for aripiprazole and placebo (a)tients to $0.0 \mathrm{~kg}$ vs. $-0.2 \mathrm{~kg}$, respectively. The proportion of patients meeting a weight gain criterion of $\geq 7 \%$ of body weight was aripiprazole ( $3 \%$ ) compared to placebo (2\%). In a 26 -week schizophrenia trial, weight change respectively, for ABILIFY (aripiprazole)- and placebo-treated patients was $-0.5 \mathrm{~kg}$ and $-0.5 \mathrm{~kg}$ for those with
$\mathrm{BM} \mid<23,-1.3 \mathrm{~kg}$ and $-0.6 \mathrm{~kg}$ for those with BMI 23 to 27 , and $-2.1 \mathrm{~kg}$ and $-1.5 \mathrm{~kg}$ for those with BMI $>27$. The percentage of AB:LLFY- and placebo-treated patients, respectively, with $\geq 7 \%$ increase in baseline body weight was $6.8 \%$ and $3.7 \%$ for those with BMI $<23,5.1 \%$ and $4.2 \%$ for those with BMl 23 to 27 and $5.7 \%$ and $4.1 \%$ for those with BMI $>27$. In a 52-week schizophrenia trial, weight change for ABilifY-treated $\mathrm{BMI}>27$. The $\mathrm{kg}$ for those with BMl $<23,1.4 \mathrm{~kg}$ for those with BMl 23 to 27 , and $-1.2 \mathrm{~kg}$ for those with for those with BMI $<23,19 \%$ for those with BMI 23 to 27 , and $8 \%$ tor those with BMI $>27$.

ECG Changes: Pooled analysis of placebo-controlled trials in patients with schizophrenia or bipolar mania reated with oral aripiprazole or in patients with agitation associated with schizophrenia or bipolar mania placebo of potentially important changes in ECG parameters. Oral aripiprazole was associated with a median increase in heart rate of 5 beats per minute compared to a 1 beat per minute increase among placebo patients. Adverse Events in Long-Term, Double-Blind, Placebo-Controlled Trials

The adverse events reported in a 26-week, double-blind trial comparing oral ABILIFY and placebo in patients with schizophrenia or bipolar mania were generally consistent with those reported in the shortOther Adverse Events Observed During the Premarketing Evaluation of Oral Aripiprazole The following adverse events were reported with oral aripiprazole at multiple doses $22 \mathrm{mg} / \mathrm{day}$ in clinical
trials $(8456$ patients, 5365 patient-years of exposure). This list may not include events previously listed elsewhere in the labeling, those events for which a drug cause was remote, those terms which were so general as to be uninformative, and those events reported with an incidence of $\leq 0.05 \%$ and which did not have a substantial probability of being acutely life-threatening. Frequent events are those occurring in at those occurring in fewer than $1 / 1000$ patients. Blood and Lymphatic System Disorders: lnfrequent anaemia, lymphadenopathy, leukopenia (including agranulocytosis, neutropenia); Rare - leukocytosis tarhycardia (including ventricular, supraventricular, sinus): infreguent - bradycardia, parpitations, cardia failure (including congestive and acute), myocardial infarction, cardiac arrest, atrial fibrillation, supraventricular), angina pectoris, cyanosis, bundle branch block (including left, right), myocardia ischaemia; Rare - atrial flutter, cardiomegaly, cardiomyopathy, cardiopulmonary failure. Ear and Labyrinth Disorders: Infrequent - ear pain, vertigo, tinnitus; Rare - deafness. Endocrine Oisorders: Infrequent -
hypothyroidism; Rare - goitre, hyperparathyroidism, hyperthyroidism. Eye Disorders: Frequent conjunctivitis: Infrequent - eye redness, eye irritation, dry eye, blepharospasm, visual disturbance, eve pain, eye discharge. blepharitis, cataract, lacrimation increased; Rare - eyelid function disorder Disorders: Frequent - lose a, photophobia, diplopia, eyelid ptosis, eye haemorrhage. Gastrointestina astritis, haemorrhoids abdominal Infrequent - flatulence, dysphagia, gastroesophageal reflux disease haemorrhage, abdominal pain lower, oral pain, retching, faecaloma, gastrointestinal haemorrhage, ulcer
(including gastric, duodenal, peptic), tooth fracture, gingivitis, lip dry; Rare - abdominal tenderness, chapped lips, periodontitis, aptyalism, gastrointestinal pain, hypoaesthesia oral, inguinal hernia, swollen tongue, colitis, haematemesis, hyperchlorhydria, irritable bowel syndrome, besophagitis, faeces hard, gingival bleeding, glossodynia, mouth ulceration, reflux oesophagitis, cheilitis, intestinal obstruction, pancreatitis, eructation, gastric ulcer haemorrhage, melaena, glossitis, stomatitis. General Disorders and
Administration Site Conditions: Frequent - asthenia, pyrexia, chest pain, gait disturbance; Infrequent -
malaise, oedema, influenza-like liliness, chills, general physical health deterioration, feeling jittery, mobility decreased, thirst, feeling cold, difficulty in walking, facial pain, sluggishness, condition aggravated; Rare inflammation localized, swelling, energy increased, inflammation, abasia, xerosis, feeling hot, hyperthermia, hypothermia. Hepatobillary Disorders: infrequent - cholecystitis (including acute and
chronic); Rase - cholelithiasis, hepatitis. Immune System Disorders; Infrequent - hypersensitivity. Imfections and infestations: Frequent - respiratory tract infection (including upper and lower), pneumonia; Infrequent - cellulitis, dental caries, vaginitis, vaginal infection, cystitis, vaginal mycosis, eye infection, gastroenteritis, onychomycosis, vaginal candidiasis, otitis media, folliculitis, Candidiasis, otitis externa,
pyelonephritis, rash pustular; Rare - appendicitis, septic shock. Injury, Poisoning, and Procedura complications: Frequent - fall, skin laceration, contusion, fracture; Infrequent - blister, scratch, joint sprain, burn, muscle strain, periorbital haematoma, arthropod bite/sting, head injury, dislocation, alcohol poisoning, road traffic accident, self mutilation, eve penetration, injury asphyxiation, poisoning, heat exhaustion, heat stroke. Investigations: Frequent - weight decreased, blood creatine phosphokinase increased; infrequent - blood glucose increased, heart rate increased, body temperature increased, haemoglobin decreased, aspartame aminotransterase increased, blood urea increased electrocardiogram ST segment abnormal (including depression, elevation), haematocrit decreased, hepatic enzyme increased, blood bilirubin increased, blood glucose decreased, blood creatinine increased, blood alkaline phosphatase increased, blood pressure decreased, blood potassium decreased, blood urine
present, electrocardiogram 0T corrected interval prolonged; Rare - transaminases increased, blood
triglycerides increased, blood uric acid increased, cardiac murmur, easiniphil count increased, neutrophil count increased, platelet count increased, red blood cell count decreased, white blood cell count decreased, white blood cells urine positive, bacteria urine identified, blood lactate dehydrogenase increased, blood potassium increased, neutrophil count decreased, urine output decreased, blood creatine heart rate decreased, increased, ECG signs of myocardial ischemia, electrocardogram T-wave inversion, glucose tolerance decreased, glycosylated haemoglobin decreased, muscle enzyme increased. Metabolism and Mutrition Disorders: Frequent - decreased appetite (including diet refusal, markedly reduced dietary intake), dehydration; Infrequent - anorexia, increased appetite, hypercholesterolaemia, hypokalaemia, hyperglycaemia, diabetes mellitus, hypoglycaemia, hyponatremia, diabetes mellitus noninsul in-dependent, hyperlipidaemia, obesity fincluding overweight), polydlpsia; Rare
hypertriglyceridaemia, gout, hypernatraemia, weight fluctuation, diabetes mellitus inadequate control. hypertriglyceridaemia, gout, hypernatraemia, weight fluctuation, diabetes mellitus inadequate control.
Musculoskeletal and Connective Tissue Disorders: Frequent - musculoskeletal pain (including neck, jaw, chest wall, bone, buttock, groin, flank, musculoskeletal chest, pubic, and sacral), muscle rigidity, muscle cramp; Infrequent - muscle twitching, joint swelling, muscle spasms, muscle tightness, arthritis,
osteoarthritis, muscular weakness, joint range of motion decreased, sensation of heaviness; Rare tendonitis, osteoporosis, trismus, arthropathy, bursitis, exostosis, night cramps, coccydynia, joint torticollis. Nervous System Disorders: Frequent - lethargy, dyskinesia; Infrequent - disturbance in attention, parkinsonism, dystonia drooling, cogwheel rigidity, dysarthria, paraesthesia, hypoaesthesia, loss attention, parkinsonism, dystonia, drooling, cogwheel rigidity, dysarthria, paraesthesia, hypoaesthesia, loss of consciousness (including depressed level of consciousness), hypersomnia, psychomotor hyperactivity, ataxia, dementia, hypotonia, burning sensation, dysgeusia, restless leg syndrome, hypertonia, Parkinson's ataxia, dementia, hypotonia, burning sensation, dysgeusia, restless leg syndrome, hypertonia, Parkinson's
disease, akinesia, dysphasia, transient ischaemic attack, facial palsy, hemiparesis, myoclonus, sciatica; disease, akinesia, dysphasia, transient ischaemic attack, facial palsy, hemiparesis, myoclonus, sciatica;
Rare - bradykinesia, coordination abnormal, cognitive disorder, syncope vasovagal, carpal tunnel syndrome, hyporeflexia, intention tremor, muscle contractions involuntary, sleep apnea syndrome, dementia Alzheimer's type, epilepsy, hyperreflexia, mastication disorder, mental impairment, nerve
compression, parkinsonian gait, tongue paralysis, aphasia, choreoathetosis, formication, masked compression, parkinsonian gait, tongue paralysis, aphasia, choreaathetosis, formication, masked lacies,
neuralgia, paresthesia oral, parkinsonian rest tremor cerebral haemorrhage, dizziness exertional, neuralgia, paresthesia oral, parkinsonian rest tremor, cerebral haemorrhage, dizziness exertional, haemorrhage. Psychiatric Disorders: Frequent - schizophrenia (including schizoaffective disorder), depression (including depressive symptom), hallucination (including auditory, visual, tactile, mixed,
olfactory, and somatic), mood altered (including depressed, euphoric, elevated, and mood swings), paranoia, irritability, suicidal ideation, confusional state, aggression, mania, delusion (including persecutory, perception, somatic, and grandeur); intrequent - tension, nervousness, nightmare, excitability, panic attack (including panic disorder, panic disorder with agoraphobia, and panic reaction), abnormal dreams, apathy, libido decreased, hostility, suicide attempt, bipolar disorder (including bipolar l), libido disorder, anger, delirium, acute psychosis, disorientation, bruxism, hypomania, obsessive-compuls fve affect, impulsive behaviour; Rare - blunted affect, cognitive deterioration, logorrhea, psychomotor agitation, social avoidant behaviour, psychomotor retardation, suspiciousness, affect lability, anorgasmia, fear, homicidal ideation, tic, premature ejaculation, dysphemia, bradyphrenia, derealisation, depersonalisation. (including acute and chronic), urinary hesitation, enuresis, nephrolithiasis, micturition urgency, polyuria: Rare - nocturia, proteinuria, glycosuria, calculus urinary, azotaemia. Reproductive System and Broast
Disorders: Infrequent - erectile dysfunction, vaginal discharge, amenorrhoea, vaginal haemorrhage, menstruation irregular, menorrhagia, premenstrual syndrome, testicular pain, genital pruritus female, ovarlan cyst, benign, prostatic hyperplasia, prostatitis; Rare - gynaecomastia, priapism (including spontaneous penile erection), breast pain, pelvic pain, epididymitis, galactorrhoea, uterine haemorrhage. Respiratory, Thoracic, and Mediastinal Disorders: Frequent - dyspnoea (including exertional); infrequentsinus congestion, rhinorrhoea, wheezing, epistaxis, asthma, hiccups, productive cough, chronic obstructive
airways disease (including exacerbated), rhinitis allergic, pneumonia aspiration, pulmonary congestion, airways disease (including exacerbated), rhinitis allergic, pneumonia aspiration, pulmonary congestion,
sinus pain, respiratory distress, dry throat, hoarseness; Rare - bronchopneumopathy, haemoptysis, respiratory arrest, sneezing, hypoxia, pulmonary embolism, pulmonary oedema (including acute), respiratory failure, brochospasm, nasal dryness, paranasal sinus hypersecretion, pharyngeal erythema, rhonchi, tonsillar hypertrophy, asphyxia, Mendelson's syndrome. Skin and Subcutaneous rissue
Disorders: Infrequent - hyperhydrosis, erythema, pruritis (including generalised), dry skin, decubitus ulcer, skin ulcer, acne, eczema, hyperkeratosis, swelling face, skin discoloration, photosensitivity reaction, skin irritation, alopecia rash maculopapular, cold sweat, scab, face oedema, dermal cyst, psoriasis, night irritation, alopecia, rash maculopapular, cold sweat, scab, face oedema, dermal cyst, psoriasis, night
sweats, rash erythematous; Rare - rash scaly, urticaria, rash maculopapular, rosacea, seborrhoea, sweats, rash erythematous; Rare - rash scaly, urticaria, rash maculopapular, rosacea, seborthoea,
periorbital oedema, rash vesicular. Vascular Disorders: Frequent - hypotension; Infrequent - het flush (including flushing), haematoma, deep vein thrombosis, phlebitis; Rare - pallor, petechiae, varicơse veiñn, other Adverse Events Observed During the Premarketing Evaluation of Ariplprazole Injection

The following adverse events were reported with aripiprazole injection at doses $z 1 \mathrm{mg} /$ day in clinical trials (749 patients). This list may not include events previousiy listed elsewhere in the labeling, those events for which a drug cause was remote, those terms which were so general as to be uninformative, and those acutely life-threatening. Frequent events are those occurring in at least $1 / 100$ patients; infrequent events are those occurring in $1 / 100$ to $1 / 1000$ patients; rare events are those occurring in fewer than $1 / 1000$ Site Conditions: Intrequent - injection site sting ing, abnormal feeling, injection site pruritus, injection site swelling, venipuncture site bruise. Infections and Infostations: Infrequent - bacteruria, urinary tract introcardiogram T-wave abnormal. Psychiatric Disorders; infrequent - intentional self-injury, Respiratory, Thoracic, and Mediastinal Disorders: Infrequent - pharyngolaryngeal pain, nasal congestion. Vascular Disorders: Infrequent - blood pressure fluctuation.

Postintroduction Reports: Reported since market introduction and temporally (not necessarily causally) related to aripiprazole theraov: allergic reaction (eq, anaphylactic reaction, angioedema, laryngospasm, oropharyngeal spasm, pruritis, or urticaria), grand mal seizure, and jaundice.

DRUG ABUSE AND DEPENDENCE: Aripiprazole is not a controlled substance.

Abuse and Dependence: Aripiprazole has not been systematically studied in humans for its potential for abuse, tolerance, or physical dependence. While the clinical trials did not reveal any tendency for any drugseeking behavior, it is not possible to predict on the basis of this limited experience the extent to which a carefully for a history of drug abuse and closely observed for signs of ABILIFY (aripiprazole) misuse or abuse. OVERDDSAGE: 76 cases of deliberate or accidental overdosage with oral ABILIFY alone or in combination with other substances were reported worldwide [44 cases with known outcome, 33 recovered without cases were in children lage 12 and younger) involving oral aripiprazole ingestions up to $195 \mathrm{mg}$ with no fatalities. The largest known acute ingestion was $1080 \mathrm{mg}$ of oral aripiprazole (36 times maximum $5 \%$ of all overdose cases) were vomiting, somnolence, and tremor. For more information on symptoms of overdose, see Full Prescribing Information.

Management of Overdosage: No specific information is available on the treatment of overdose with aripiprazole. An electrocardiogram should be obtained in case of overdosage and, if QTc interval prolongation is present, cardiac monitoring should be instituted. Otherwise, management of overdose should concentrate on supportive therapy, maintaining an adequate airway, oxygenation and ventilation, and management of
symptoms. Close medical supervision and monitoring should continue until the patient recovers. Charcoal: in the event of an overdose of ABILIFY, an early charcoal administration may be useful in partially preventing the absorption of aripiprazole. Administration of $50 \mathrm{~g}$ of activated charcoal, one hour after a single 15 -mg oral dose of aripiprazole, decreased the mean AUC and $\mathrm{C}_{\text {max }}$ of ariptprazole by $50 \%$. Hemodialysis: Although
there is no information on the effect of hemodialysis in treating an overdose with aripiprazole, hemodialysis is there is no information on the effect of hemodialysis in treating an overdose with aripiprazole, hemodh
unlikely to be useful in overdose management since aripiprazole is highly bound to plasma proteins.

Tablets manufactured by Otsuka Pharmaceutical Co., Ltd., Tokyo, 101-8535 Japan or Bristol-Myers Squiblo Orally Disintegrating Tablets, Oral Solution and Injection manufactured by Bristol-Myers Squibb Company,

Distributed and marketed by Otsuka America Pharmaceutical, Inc., Rockville, MD 20850 USA

Marketed by Bristol-Myers Squibb Company, Princeton, NJ 08543 USA

Bristol-Myers Squibb Company

Otsuka America Pharmaceuntical, Inc.

Princeton, NI 08543 U.S.A.

Rockvillo, MD 20850 U.S.A.

○ 2007, Otsuka Pharmaceutical Co. Ltd., Toky0, 101-8535 Japan 